\title{
La mise en nourrice, une pratique répandue en France au XIX siècle
}

\section{Emmanuelle ROMANET}

\section{(2) OpenEdition}

\section{Journals}

Édition électronique

URL : http://journals.openedition.org/transtexts/497

DOI : $10.4000 /$ transtexts.497

ISSN : 2105-2549

Éditeur

Gregory B. Lee

Référence électronique

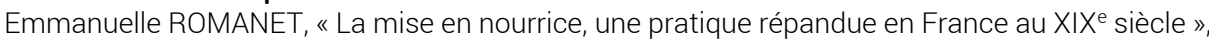
Transtext(e)s Transcultures 跨文本跨文化 [En ligne], 8 | 2013, mis en ligne le 02 décembre 2013, consulté le 30 avril 2019. URL : http://journals.openedition.org/transtexts/497 ; DOI : 10.4000/ transtexts.497 


\title{
Transtext(e)s
}

Transcultures 跨文本跨<smiles>[Mg]</smiles>

Journal of Global Cultural Studies

8 | 2013 :

Genre et filiation : pratiques et représentations

Pères, mères, nourrices

\section{La mise en nourrice, une pratique répandue en France au XIXe siècle}

\author{
EMMANUELLE ROMANET
}

\section{Résumés}

Français English

La mise en nourrice des enfants des villes à la naissance est une réalité en France au XIXe siècle. Le modèle de la mère au foyer qui s'occupe de ses enfants n'est devenu effectivement la référence dominante qu'au XXe siècle. Cette pratique qui a des conséquences démographiques, est ancienne, imposée à la mère et touche tous les milieux sociaux. Elle nous en dit long sur l'organisation de la famille et les soins apportés à l'enfant, comme nous le montre le cas de Lyon, ville industrielle dans laquelle la mise en nourrice est une pratique très ancienne et très répandue. Lyon illustre, à merveille, l'allaitement mercenaire.

Newborn babies were commonly entrusted to wet nurses and taken care of out of home in $19^{\text {th }}$ century France. The model of at home housewives caring for their children only became a dominant pattern in the 2oth century. This ancient practice was imposed on mothers across all social categories and classes and had major demographical consequences. It was very relevant for the organization of the family unit and childcare, and the industrial city of Lyon, where fostering of newborns was a very old and traditional practice, is a good illustration of that phenomenon. Lyon is a prime example of mercenary breast-feeding.

\section{Texte intégral}




\section{Etat des lieux d'une pratique répandue en France au XIXe siècle}

1 D'après la définition du dictionnaire, la mise en nourrice est « l'allaitement des nouveaux-nés par une femme requise à cet effet ... $\gg^{1}$ Cette pratique a toujours existé : le code d'Hammurabi ${ }^{2}$ fixait déjà les devoirs des nourrices. En France, un édit du XIVe siècle fixe même la rémunération de celles de la région parisienne. $^{3}$

2 Cette pratique s'est développée à partir du XVIIe siècle et ce, dans les centres urbains en priorité. Elle s'accentue au XVIIIe siècle. A Paris en 1780, par exemple, sur les 21000 enfants nés cette année-là, seuls 1 ooo enfants sont nourris par leur mère. Au XIXe siècle, pour les années 1887-1888, le nombre d'enfants mis en nourrice hors de la ville pour 100 naissances est de 2,8 pour Lille ; 9,6 pour Bordeaux ; 29,3 pour Paris et 47,9 pour Lyon. ${ }^{4}$ Ces chiffres illustrent une pratique très largement répandue.

3 Cette pratique est tellement importante, que tout une infrastructure a été mise en place pour l'encadrer. On peut même parler d'un véritable marché. Des Bureaux de placement des nourrices sont créés : les nourrices viennent à Paris et y restent parfois trois semaines à leurs frais, en attendant d'être choisies. Parfois, elles repartent sans nourrisson et après y avoir abandonné le leur. C'est le cas lorsque les milieux ruraux rencontrent des difficultés (crises) ; les femmes viennent alors proposer leurs services (leur lait) à Paris.

$4 \quad$ Les médecins-accoucheurs ou les sages-femmes mettent en contact les mères qui viennent d'accoucher et les « rabatteurs » qui proposent des nourrices. Médecins et sages-femmes reçoivent, au passage, leurs commissions.

$5 \quad$ Toutes les classes de la société recourent à l'allaitement mercenaire et il y a une relation entre les moyens financiers des parents et la « qualité » de la nourrice. Plus la famille est pauvre, plus le nourrisson est expédié loin. Quant aux enfants abandonnés, ils sont placés par les institutions parfois très loin et dans des conditions atroces. Les enfants illégitimes (hors mariage) représentent 25 à $28 \%$ des naissances à Paris dans les années $1860 .{ }^{5}$ Ils sont abandonnés car leurs mères ne peuvent pas les assumer, sur le plan matériel comme sur le plan social.

Dans la deuxième moitié du XIXe siècle, presque toutes les familles aisées ont une nourrice chez elles. Le phénomène s'est généralisé à partir de 1830 ; il est le fait d'une nouvelle bourgeoisie (d'affaires et d'industrie) qui aime paraître. La nourrice « sur lieu » devenant alors un signe extérieur de richesse. C'est une pratique que l'on peut aussi rapprocher du logement Haussmannien. Bien entendu, cette bourgeoisie a ses propres réseaux pour trouver la nourrice idéale. Il y a donc un lien entre classes sociales et « choix » de la nourrice. Si toutes les classes sociales y ont recours, elles ne s'adressent pas au même type de nourrices.

7 La littérature française du XIXe siècle décrit cette pratique. Par exemple, dans Madame Bovary de Flaubert (1857), Emma Bovary met sa fille en nourrice chez la mère Rollet. Berthe, comme la plupart des enfants de l'époque et de sa condition est placée en nourrice. ${ }^{6}$

8 Les conséquences de cette mise en nourrice sont diverses. Première conséquence : une surmortalité infantile très élevée. Les chiffres de la mortalité des enfants placés en nourrice sont édifiants. Pour preuve, ces chiffres issus 
d'un mémoire de 1866 que le ministre de l'instruction publique (Victor Duruy) a communiqués à l'Académie de médecine.

Ce mémoire est l'oeuvre du Docteur Monot, médecin du canton de Montsauche dans la Nièvre. Il décrit le vide laissé dans les villages par le départ des femmes venues se placer comme nourrice à domicile à Paris. Entre 1858 et 1864 sur les 2884 femmes ayant accouché dans les 10 communes du canton, 1897 d'entre elle, soit les deux tiers, ont émigré. ${ }^{7}$ Le Docteur Monot évoque la mort massive des enfants nouveaux-nés laissés par leur mère. Le sort des "Petits Paris », ces nouveaux-nés de Paris mis en nourrice dans le canton, est encore pire : leur taux de mortalité atteint $71 \%$ pendant la première année de leur existence. ${ }^{8}$ Alors que le taux de mortalité infantile pour l'ensemble de la France en 1865 est de $17,9 \% .^{9}$

10 Les médecins et les moralistes s'emparent de cette question et critiquent la mise en nourrice.

11 Le mémoire du Docteur Monot va susciter un long débat (dix séances) à l'Académie de médecine. ${ }^{10}$

12 Cette prise de conscience aboutira à la loi Roussel de 1874 qui stipule que : « Tout enfant, âgé de moins de deux ans, qui est placé moyennant salaire en nourrice, en sevrage ou en garde hors du domicile de ses parents, devient, par ce fait, l'objet d'une surveillance de l'autorité publique, ayant pour but de protéger sa vie et sa santé. $»^{11}$

13 Mais quelles sont les raisons d'une telle surmortalité ? Elles sont multiples. D'alord les aléas du transport des enfants, conduits loin de chez eux par des « meneurs » qui s'en occupent mal. Ils laissent les bébés dans le froid ... Comme le raconte Emile Zola dans son ouvrage « Fécondité » (1899): «Puis il fallait voir dans les trains, quel entassement de pauvres êtres, qui criaient de faim. L'hiver surtout par les grandes neiges ; ça devenait pitoyable, tant ils grelotaient, bleus de froid, à peine couverts de maillots en loques. Souvent il en mourait ... ». ${ }^{12} \mathrm{Il}$ y a également les nourrices qui allaitent parfois aussi leur propre enfant : elles ont alors moins de lait pour l'enfant placé. Elles recourent alors à l'allaitement artificiel (lait d'animaux, ...) ou à des bouillies ; une alimentation inadaptée pour des nourrissons. Sans compter les mauvaises conditions d'hygiène de la vie paysanne (cohabitation avec les animaux, fumier ...) ou le manque de surveillance (elles vont au champs, ramassent du bois ... et laissent le bébé seul à la maison). Elles soignent elles-mêmes l'enfant quand il est malade au lieu d'appeler le médecin.

Le panier à porter les enfants, gravure du XIXe siècle. 


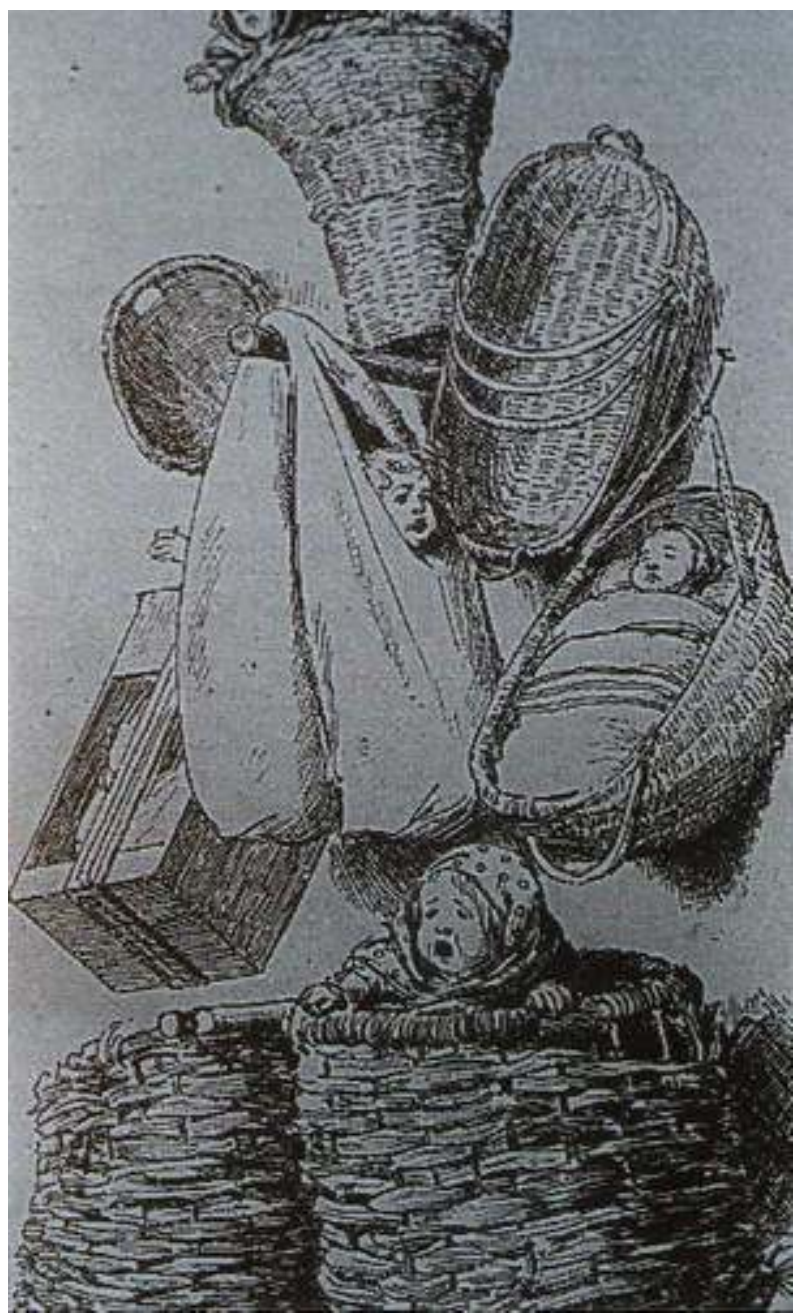

Deuxième conséquence de cette mise en nourrice : un taux de natalité élevé. N'allaitant plus, les femmes sont moins longtemps protégées contre les grossesses rapprochées. C'est le cas à Lyon, où le placement en nourrice est courant. Cette pratique a pour conséquence directe de rendre les épouses très fécondes : la moyenne calculée dans le milieu des ouvriers en soie de Lyon atteint plus de huit enfants par femme. Le tiers des femmes donnant même naissance à dix enfants et plus ! Le milieu des bouchers était encore plus prolifique, puisque les femmes accouchaient tous les ans $!^{13}$

\section{Pourquoi une telle pratique?}

Il y a une reproduction de ce qui se fait depuis longtemps. La mise en nourrice des enfants de l'aristocratie s'est toujours pratiquée. Comme l'explique en 1859 Louis de Bonald, maître à penser de la noblesse française : « l'allaitement est une fonction trop animale pour une dame de qualité. » (...) Celle-ci « doit à ses enfants de tous autres services, imposant la distance et le respect. ${ }^{14}$ La bourgeoisie urbaine imite l'aristocratie et envoie, elle aussi, ses enfants en nourrice à la campagne.

16 Pourtant au XVIIIe siècle, les philosophes et les médecins s'insurgent d'une telle pratique et de ses conséquences. Ils se mobilisent, prêchant pour l'allaitement maternel. Les philosophes accusent les nourrices mercenaires d'être sales et ignorantes. Ils s'en prennent aussi au modèle aristocratique. 
Pour eux, la grande dame qui refuse son lait à son enfant « trahit la nature ». ${ }^{15}$ Et c'est Rousseau qui part en croisade pour l'allaitement maternel avec son ouvrage « Emile ou De l'éducation » (1762) : «Celle qui nourrit l'enfant d'une autre au lieu du sien est une mauvaise mère : comment sera-t-elle une bonne nourrice ? (...) Mais que les mères daignent nourrir leurs enfants, les mœurs vont se réformer d'elles-mêmes... $»^{16}$

Mais les Lumières n'ont pas ou peu changé les choses dans ce domaine. Il y eut bien la " mode de la mamelle », comme le relate Madame de Genlis (1746-1830), femme de lettres adepte d'une pédagogie rousseauiste et en charge de l'éducation des enfants d'Orléans, dont le futur Louis-Philippe : " ...des nobles dames de la Cour se faisaient apporter leurs bébés pour les allaiter... ${ }^{17}$ Mais la mode passa. Les idées des Lumières sur le sujet vont également amener à une certaine prise de conscience chez les femmes de la bourgeoise : si elles n'allaitent toujours pas elles-mêmes leurs enfants, elles ont compris l'importance de surveiller les nourrices et donc de les avoir sous leur toit.

C'est ainsi qu'à partir de la seconde partie du XIXe siècle, la grande et moyenne bourgeoisie fait venir la nourrice " sur-lieu », soit au domicile des parents. Elle est un signe extérieur de richesse pour ses patrons. Elle est donc bien habillée et choyée. Elle a une place à part dans la domesticité. Elle est bien payée, mais elle fait des sacrifices : elle a quitté sa famille. Elle s'est présentée au bureau des nourrices de la ville avec son bébé. C'est un bureau qui fonctionne comme un marché aux bestiaux : le docteur palpe ses seins, goûte son lait, flaire son haleine... Après l'embauche, elle doit renvoyer son enfant qu'une « meneuse » raccompagne jusqu'au village.

\section{Bureaux de nourrices, Paris.}

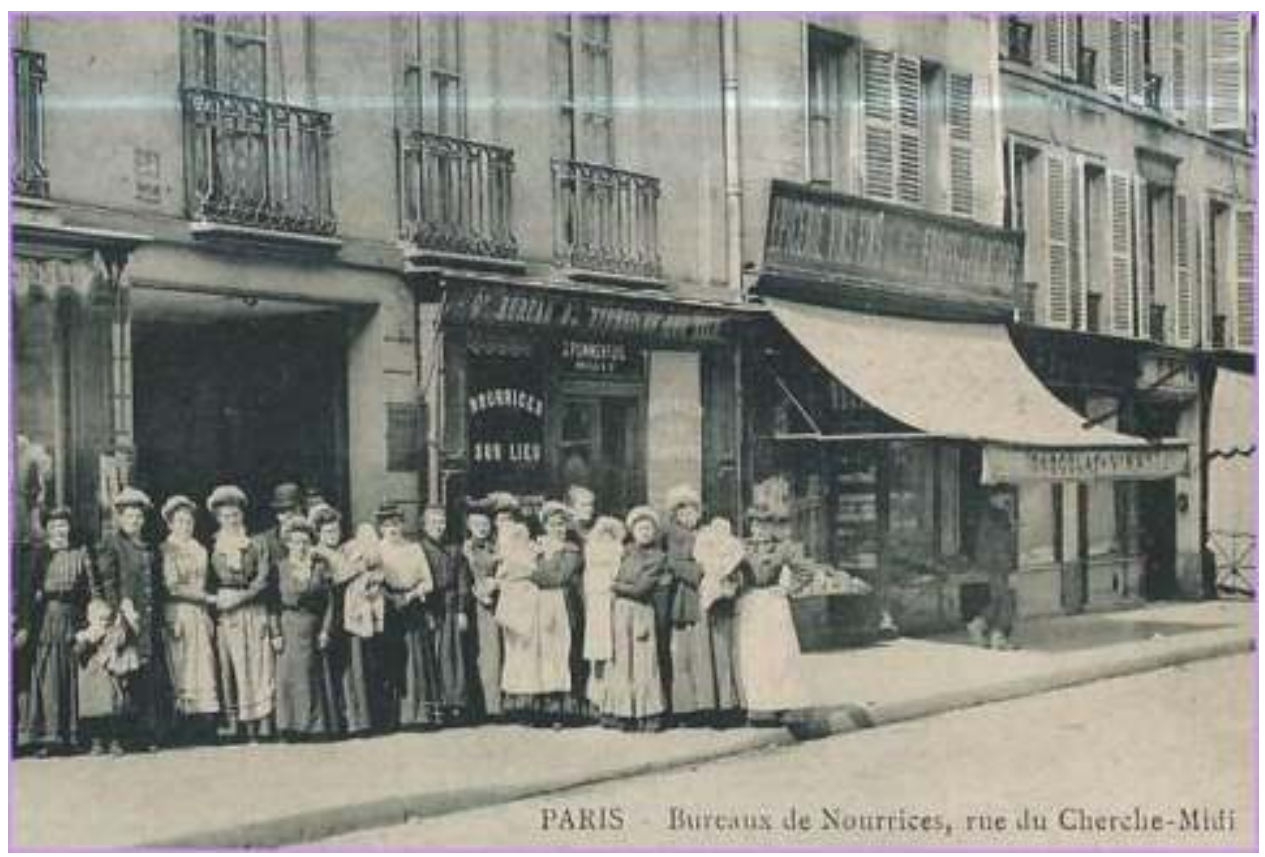

Cette pratique touche tous les milieux sociaux. L'aristocratie et la bourgeoisie urbaine entendent préserver le rôle de représentation sociale de la femme. C'est à dire, les mondanités. Il faut en outre prendre en compte d'autres données : le caractère inesthétique de l'allaitement qui passe, à l'époque, pour gâter la beauté des seins, et les lourdeurs d'un tabou selon lequel les relations sexuelles sont proscrites au cours de cette période. Déjà pour les médecins de l'Antiquité 
(comme Soranos d'Ephèse, médecin grec du début du IIe siècle), une femme ne peut pas accomplir à la fois ses devoirs d'épouse et ses devoirs de mère nourricière. ${ }^{18}$ L'avénement du christianisme a fait apparaitre de nouvelles raisons d'éloigner le nourrisson de sa mère. Le mariage chrétien impose à l'époux et à l'épouse la fidélité réciproque. Or le tabou persiste qui interdit les rapports sexuels pendant l'allaitement. Donc si le mari ne veut pas commettre l'adultère, il va envoyer son enfant en nourrice. ${ }^{19}$

Dans les milieux populaires, notamment ouvriers, c'est la nécessité du travail des femmes qui pousse les couples à mettre leurs enfants en nourrice. De leur côté, les femmes d'artisans et de commerçants aident leurs maris : elles tiennent la boutique, l'échoppe.

Cette décision semble imposée par les hommes. Un médecin du XVIIe siècle raconte le cas d'une de ses patientes, qui ayant perdu deux enfants en nourrice, mit au monde une petite fille et demanda à son mari l'autorisation de la nourrir. Quand la mère allaite, il est admis que la première dent du bébé légitime le sevrage : le mari réclame alors ses droits. ${ }^{20}$ Pour Yvonne Knibiehler, qui a travaillé sur l'histoire de la maternité, « la maternité n'appartient pas aux femmes ${ }^{21}$ et pour Françoise Héritier ${ }^{22}$, les hommes se sont toujours efforcés, ils s'efforcent encore, consciemment ou non, de contrôler la procréation dans son ensemble, et donc aussi l'allaitement. Selon les moments, les lieux, les milieux, ils empêchent les femmes d'allaiter ou bien ils les y contraignent.

L'allaitement ne dépend donc pas seulement du désir de la mère. Celle-ci est soumise à des normes et à des codes. Des pressions s'exercent sur elle de la part de son mari, de son médecin, ou de son entourage.

\section{La situation à Lyon au XIXe siècle}

A Lyon, en 1890, plus de la moitié des enfants nés sont confiés à des nourrices « à emporter » (4 203 sur 8101 naissances), sans compter les bébés (917) pourvus d'une nourrice "sur lieu ${ }^{23}$ Rappelons-nous les chiffres déjà évoqués : dans les années 1887-1888, pour 100 naissances : 47,9 petits Lyonnais vont en nourrice hors de la ville contre 2,8 petits Lillois. ${ }^{24}$

Lyon est un cas particulier. Ville industrielle, elle se distingue des autres villes françaises. L'allaitement mercenaire y est très ancien (dès le XVIIe siècle) et touche presque toutes les classes sociales. Cette pratique, ailleurs réservée aux classes aisées de la population urbaine, est ici observable dans les familles bourgeoises comme dans celles d'artisans (bouchers ou menuisiers) ; mais aussi chez les ouvriers et ouvrières en soie. Les raisons qui les poussent à envoyer leurs enfants à la campagne ne sont bien évidemment pas les mêmes. Pour les familles aisées " ne pas nourrir est la marque d'un rang social élevé ». ${ }^{25}$ Quant aux femmes d'artisans, elles travaillent près de leurs maris ou dans la boutique et ne peuvent s'occuper des enfants. Tout comme les femmes de canuts. Il y a un lien entre travail de la femme et mise en nourrice : la nouvelle dynastie industrielle des Gillet dans la première moitié du XIXe siècle, en est un bon exemple : "François et son épouse travaillent beaucoup. Et le couple doit mettre ses enfants en nourrice. " A la génération suivante, les femmes ne travaillent plus et les enfants restent au foyer. ${ }^{26}$ Joséphine, l'épouse d'Antoine Lumière aide son mari à l'atelier de photographie puis à l'usine de Montplaisir, comme le raconte leur fils Auguste Lumière. ${ }^{27}$ Ce n'est pas 
l'obligation de travailler qui pousse la bourgeoise lyonnaise à mettre son enfant en nourrice. Il faut au préalable, faire remarquer que la bourgeoisie lyonnaise est catholique et très féconde. Cela a un impact sur ces questions dites de nourrissage. Les dames de la bonne bourgeoisie lyonnaise nourrissent quand elles le peuvent. Mais fatiguées par des grossesses rapprochées, nombre d'entre elles y renoncent à partir du troisième ou du quatrième enfant. Et les médecins les encouragent dans ce sens. ${ }^{28}$ On sait que le malthusianisme bourgeois qui sévit à Paris au XIXe siècle ne se retrouve pas à Lyon. Il n'est pas rare de voir des familles de dix ou douze enfants. La fertilité est une tradition familiale qui s'explique par la religion et la nécessité de l'avenir dynastique.

La bourgeoise lyonnaise, bien qu'elle ne travaille pas, ne peut pas s'occuper de sa progéniture. Elle a une vie mondaine. Les femmes reçoivent un après-midi par semaine. Et quand elles ne reçoivent pas, elles sont reçues. Les annuaires du « Tout Lyon » mentionnent en face du nom de famille, le jour de réception hebdomadaire.

Le choix d'une nourrice : La grossesse est une période pendant laquelle la bourgeoise doit organiser le devenir matériel de son enfant. Il lui faut choisir une nourrice. Ce qui n'est pas facile. Mais les couples disposent de conseils donnés par les médecins ou les moralistes, qui, faute de ne pouvoir faire cesser cette pratique, les aident dans leur choix. Il y a des critères médicaux (hygiène physique) et des critères qui relèvent davantage de la symbolique sociale comme l'haleine, la taille des seins ou la couleur des cheveux ; les rousses étant censées avoir un lait aigre. On peut remarquer que les conseils médicaux n'ont pas vraiment évolué depuis l'Antiquité. Ils sont tirés de l'ouvrage du médecin Soranos d'Ephèse, qui constitue la somme de tout le savoir antique sur cette question.

Ces conseils pour choisir une nourrice on les retrouve à quelques détails près dans de très nombreux ouvrages médicaux jusqu'au milieu du XIXe siècle. ${ }^{29} \mathrm{La}$ famille peut aussi se faire recommander une nourrice dans le village où elle possède sa maison de campagne ; ces propriétés n'étant jamais très éloignées de Lyon. La nourrice " sur-lieu » est très surveillée au sein de la maisonnée. Dans une correspondance privée, on apprend que Marthe, une bourgeoise lyonnaise, doit se séparer de sa nourrice « qui siffle les bouteilles et n'a pas non plus assez de lait $\gg 3^{30}$ On retrouve les nourrices des familles aisées sur les grandes places de Lyon. Elles viennent y promener les enfants. Ce sont des espaces très prisés de la bonne société. Un contemporain décrit le spectacle qu'offre la place Bellecour l'après-midi: "L'aspect de Bellecour à cette heure est une harmonie de notes claires où domine le blanc des petits manteaux, le blanc des robes longues des nouveaux-nés, celui des tabliers et des manches des bonnes; puis, c'est le bleu et le rouge vif des robes des fillettes (...) c'est le rouge cerise des rubans-étendards qui ornent si étrangement les manteaux des nourrices (...) Enfin, toute la gamme tendrement nuancée des ombrelles, qui, de loin, transforment Bellecour en un parterre mouvant de fleurs phénoménales." ${ }^{1}$

Place Morand : les nounous, leurs landeaux et le kiosque à musique un après-midi d'hiver (BML / PO546 SA 17/16 Fonds Jules Sylvestre. Auteur: Jules Sylvestre). 


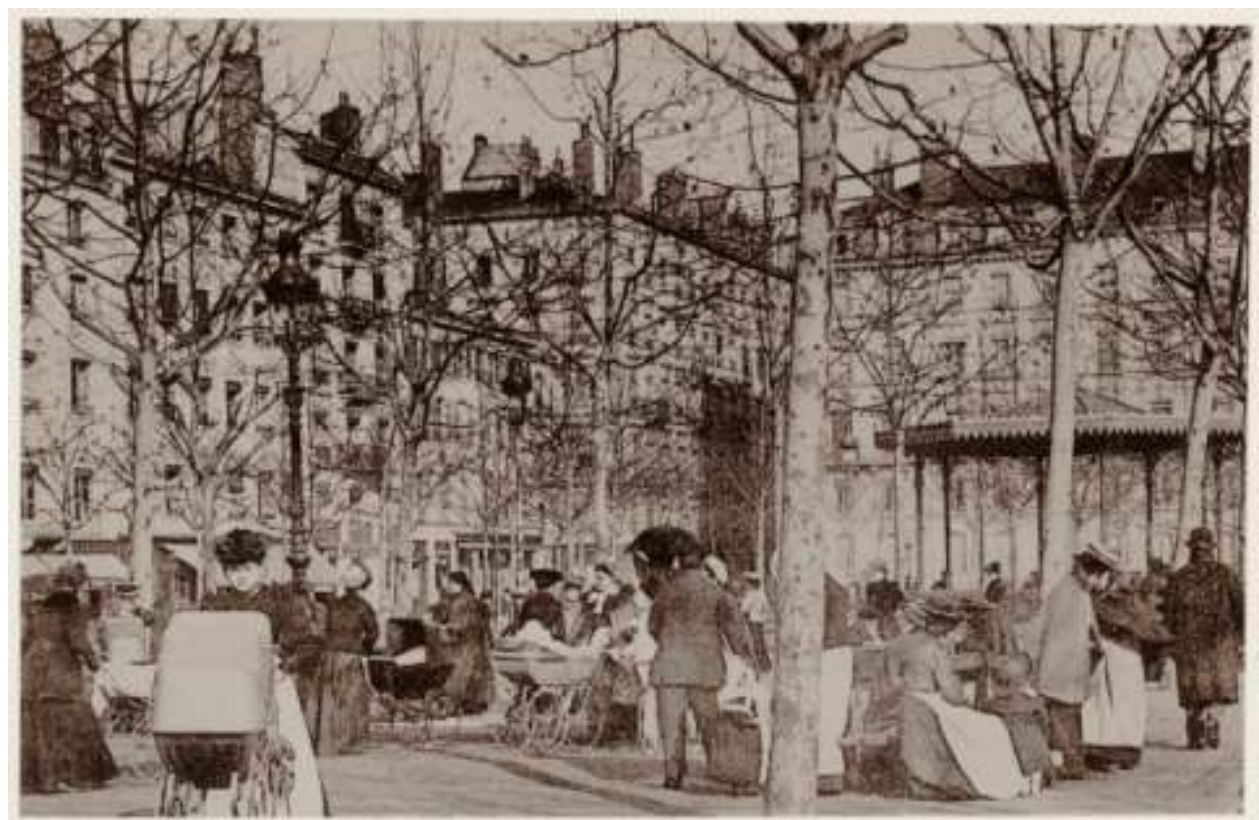

Quelle est la place de l'homme dans la décision de mettre un enfant en nourrice ? Bernadette Angleraud et Catherine Pellissier expliquent que la maternité au XIXe siècle est considérée comme la vocation naturelle et exclusive de la femme. La naissance et la prime éducation sont traditionnellement considérées comme " une affaire de femmes ». Cependant les sources intimes (journaux, correspondances) contrarient cette idée reçue. Les pères lyonnais se montrent très attentifs à ces questions. $»^{32}$ Mais si la nourrice " sur lieu » est réservée à l'élite, les couples d'artisans ou d'ouvriers s'adressent plutôt aux nourrices à emporter. Plus la somme allouée à la nourrice est élevée, moins l'enfant sera loin de Lyon. Les particuliers ne sont pas les seuls à choisir de mettre en nourrice, à la campagne, les enfants nouveaux nés ou en bas âge. A Lyon, les hôpitaux de la Charité et de l'Hôtel Dieu, puis à partir de 1869 le département du Rhône, sont en charge des enfants illégitimes et des enfants abandonnés et « exposés ». Ces institutions se tournent aussi vers les campagnes pour les y placer. A Lyon, le nombre annuel d'enfants admis dépasse le seuil des 2 ooo en 1842 (2 o8o exactement). Et ce sont près de 12 ooo enfants qui sont à la charge des Hospices civils de Lyon en 1838. Ils sont placés dans le Bugey, le Revermont et le Vivarais. ${ }^{33}$ L'institution ayant laissé d'importantes archives, on peut suivre les enfants placés par les Recteurs des hôpitaux puis par le département. Grâce aux registres de placements, il est possible de retrouver les familles nourricières. Par exemple : On peut lire sur le Registre de réception de la Charité des années 1825-1826 que "Joseph Monin, garçon né aujourd'hui céans illégitime de Louise Monin, agée de 24 ans de Monceveroux, Isère, domestique à Lyon, cour Ste Elisabeth chez la veuve Gay (...) A été batisé dans cet hôpital et enregistré à la mairie de cette ville. Le 7 mai 1826 . ${ }^{34}$ Le petit Joseph est très vite envoyé en nourrice. On peut le suivre grâce au Registre des enfants placés à la campagne 1826-1827 : "Joseph Monin, ${ }^{\circ} 39130$ né le 7 mai 1826, remis à Philibert Girardet et Marie Anne David au bourg de Virieux le Petit (Belley, Ain) le 8 mai 1826. $»^{35}$ On peut multiplier les exemples de ces enfants abandonnés, exposés puis placés en nourrice : Auguste Tracol est né le 6 février 1866 à l'Hospice et abandonné le 8 février. Le registre explique qu'il est « admis aux assistés, vu la vie vagabonde de sa mère qui se prostitue avec le premier venu. ${ }^{36}$ Cunégonde 
Milil, née le 28 février 1866, exposée et trouvée rue de la Charité au n ${ }^{\circ} 6$ à 8 heures et demi du soir et admise à l'hospice le 2 mars $1866 .{ }^{37}$ L'espérance de vie de ces enfants est courte. D'après François-Marguerite Barrier, Chirurgien de l'Hôtel-Dieu de Lyon, dans un article de 1866 intitulé « Note sur les moyens d'améliorer les conditions des enfants en nourrice ", à Lyon, sur les 6000 enfants placés en nourrice par an, 4 ooo décèdent. ${ }^{38}$

\section{Conclusion}

La mise en nourrice est donc une pratique répandue en France au XIXe siècle qui permet de répondre à nos interrogations sur ce qu'est la filiation. Elle montre bien que la mère au foyer qui se consacre à ses enfants est marginale pour ne pas dire inexistante au XIXe siècle. Cette pratique renvoie à d'autres questionnements comme le travail des femmes (des nourrices, mais de certaines mères aussi) : l'activité de nourrice " sur place ", permet à de nombreuses familles de paysans d'améliorer leur niveau de vie grâce au travail de ces femmes. Ce pan de l'histoire du nourrissage est bien connu pour le XIXe siècle, avec les nourrices du Morvan très recherchées à Paris et dont le travail a permis l'achat de biens, qu'il s'agisse d' un pré, d'un bois, d'un champ ou d'une maison. D'ailleurs, les maisons acquises avec l'argent gagné par les femmes de ces familles paysannes portent le nom évocateur de « maisons de lait ».

Cette pratique questionne aussi sur l'exploitation d'une classe sociale par une autre ou encore la marchandisation du corps de la femme (la nourrice) qui vend ce qui vient de son corps (son lait).

\section{Bibliographie}

Angleraud Bernadette et Pellissier Catherine, Les dynasties lyonnaises Des Morin-Pons aux Mérieux, du XIXe siècle à nos jours, Paris, Perrin, 2003.

Brunet Guy, Aux marges de la famille et de la société : filles-mères et enfants assistés à Lyon au 19 è siècle, Paris, L'Harmattan, 2008.

David Michel, « François-Marguerite (1813-1870) Chirurgien major de l'Hôtel-Dieu de Lyon, précurseur de la pédiatrie lyonnaise », Site d'enseignement d'Histoire de la médecine, Université Claude Bernard Lyon 1.

Fauve-Chamoux Antoinette, "Innovation et comportement parental en milieu urbain (XV-XIXe siècles) », Annales, Economies, Sociétés, Civilisations, nº $5,1985$.

Faÿ-Sallois Fanny, Les nourrices à Paris au XIXe siècle, Paris, Payot, 1997.

Flaubert Gustave, Madame Bovary, Paris, le livre de poche, 1999.

Fouquet Catherine et Knibiehler Yvonne, Histoire des mères du Moyen-âge à nos jours, Paris, Hachette, 1982.

Gélis Jacques, Laget Mireille et Morel Marie, Entrer dans la vie. Naissances et enfances dans la France traditionnelle, Paris, Gallimard, 1978.

Héritier Françoise, Masculin/Féminin II Dissoudre la hiérarchie, Paris, Odile Jacob, 2002.

Knibiehler Yvonne, « L'allaitement et la société », Recherches féministes, vol.16, $\mathrm{n}^{\circ} 2$, 2003.

Monot Charles, De l'industrie des nourrices et de la mortalité des petits enfants, Paris, 1867.

Monot Charles, De la mortalité excessive des enfants la première année de leur 
existence, ses causes et des moyens de la restreindre, Paris, 1872.

Pellissier Catherine, La vie privée des notables lyonnais au XIXe siècle, Lyon, Editions lyonnaises d'Art et d'Histoire, 2001.

Rollet Catherine, Histoire de l'allaitement en France : pratiques et représentations, www.santeallaitementmaternel.com.

Rollet Catherine, « Allaitement, mise en nourrice et mortalité infantile en France à la fin du XIXe siècle », Population, $\mathrm{n}^{\circ}$ 6, 1978.

Rollet-Echalier Catherine, La politique à l'égard de la petite enfance sous la IIIe République, INED-PUF, 1990.

Rousseau Jean-Jacques, Emile ou De l'éducation, livre premier.

Vingtrinier Emmanuel, La vie lyonnaise, autrefois, aujourd'hui, Lyon, Editions de Lyon, 1898.

Zola Emile, Fécondité, Bibliothèque électronique du Québec.

\section{Notes}

1 Dictionnaire Larousse, 2005.

2 Texte juridique Babylonien du 18è siècle avant $\mathrm{J}-\mathrm{C}$.

3 Nourrices et hôpitaux à Lyon au 18è siècle, Guichet du Savoir 26/07/2005, Bibliothèque municipale de Lyon.

4 Statistiques sanitaires des villes de France et d'Algérie années 1887-1888, cité par Catherine Rollet, " Allaitement, mise en nourrice et mortalité infantile en France à la fin du 19 è siècle » Population, ${ }^{\circ}$ 6, 1978, p. 1198.

5 Catherine Rollet-Echalier, La politique à l'égard de la petite enfance sous la IIIe République, Paris, PUF, 1990, p.62.

6 Gustave Flaubert, Madame Bovary, Paris, le livre de poche, 1999, p. 175.

7 Charles Monot, De l'industrie des nourrices et de la mortalité des petits enfants, Paris, 1867, p.36.

8 Charles Monot, De la mortalité excessive des enfants la première année de leur existence, ses causes et des moyens de la restreindre, Paris, 1872, p.27.

9 Jacques Gélis, Mireille Laget et Marie Morel, Entrer dans la vie. Naissances et enfances dans la France traditionnelle, Paris, Gallimard, 1978, p. 186.

10 Fanny Faÿ-Sallois, Les nourrices à Paris au 19è siècle, Paris, Payot, 1997, p. 67.

11 Fanny Faÿ-Sallois, Les nourrices à Paris au 19è siècle, op. Cit. p. 88.

12 Emile Zola, Fécondité, Bibliothèque électronique du Québec, p.448.

13 Catherine Rollet, Histoire de l'allaitement en France : pratiques et représentations, www.santeallaitementmaternel.com, p.5.

14 Yvonne Knibiehler, "L'allaitement et la société », Recherches féministes, vol. 16, $\mathrm{n}^{\circ} 2,2003, \mathrm{p} .19$.

15 Yvonne Knibiehler, «L'allaitement et la société », op. Cit. p.21.

16 Jean-Jacques Rousseau, Emile ou De l'éducation, livre premier, Les classiques des sciences sociales Université du Québec, http://classiques.uqac.ca/classiques /Rousseau_jj/emile/emile.html, p.16.

17 Yvonne Knibiehler, "L'allaitement et la société », Recherches féministes, vol. 16, $\mathrm{n}^{\circ} 2,2003, \mathrm{p} .22$.

18 Yvonne Knibiehler, "L'allaitement et la société », Recherches féministes, vol. 16, $\mathrm{n}^{\circ} 2,2003, \mathrm{p} .15$.

19 Yvonne Knibiehler, «L'allaitement et la société », op. Cit., p. 17.

20 Yvonne Knibiehler, « L'allaitement et la société », op. Cit., p.17.

21 Yvonne Knibiehler, « L'allaitement et la société », op. Cit., p. 29. 
22 Françoise Héritier, Masculin/Féminin II Dissoudre la hiérarchie, Paris, Odile Jacob, 2002.

23 Catherine Rollet-Echalier, La politique à l'égard de la petite enfance sous la IIIe République, Paris, PUF, 1990, p.82.

24 Statistiques sanitaires des villes de France et d'Algérie années 1887-1888, cité par Catherine Rollet, « Allaitement, mise en nourrice et mortalité infantile en France à la fin du XIXe siècle », Population, nº6, 1978, p. 1198.

25 Antoinette Fauve-Chamoux, "Innovation et comportement parental en milieu urbain (XV-XIXe siècles) », Annales, Economies, Sociétés, Civilisations, 1985, n 5 , pp. 1025 .

26 Bernadette Angleraud et Catherine Pellissier, Les dynasties lyonnaises : Des Morin-Pons aux Mérieux, du XIXe siècle à nos jours, Paris, Perrin, 2003, p.357.

27 Bernadette Angleraud et Catherine Pellissier, Les dynasties lyonnaises : Des Morin-Pons aux Mérieux, op. Cit., p. 490.

28 Catherine Pellissier, La vie privée des notables lyonnais au XIXeme siècle, Lyon, Editions lyonnaises d'Art et d'Histoire, 2001, p. 194.

29 Yvonne Knibiehler, «L'allaitement et la société », op. Cit., p. 15.

30 Catherine Pellissier, La vie privée des notables lyonnais au XIXème siècle, op. Cit., p. 194.

31 Emmanuel Vingtrinier, La vie lyonnaise, autrefois, aujourd'hui, Lyon, Editions de Lyon, 1898, p. 27-28.

32 Bernadette Angleraud et Catherine Pellissier, Les dynasties lyonnaises : Des Morin-Pons aux Mérieux, op. cit., p. 490.

33 Guy Brunet, Aux marges de la famille et de la société : filles-mères et enfants assistés à Lyon au 19è siècle, Paris, L'Harmattan, 2008, p.37.

34 AML CH_4Q85, Registre de réception 1825-1826.

35 AML CH_4Q262, Registre de placement 1826-1827.

36 AML CH_4Q179.

37 AML CH_4Q179.

38 Site d'enseignement d'Histoire de la médecine, Université Claude Bernard Lyon 1, Diaporama Michel David.

\section{Table des illustrations}

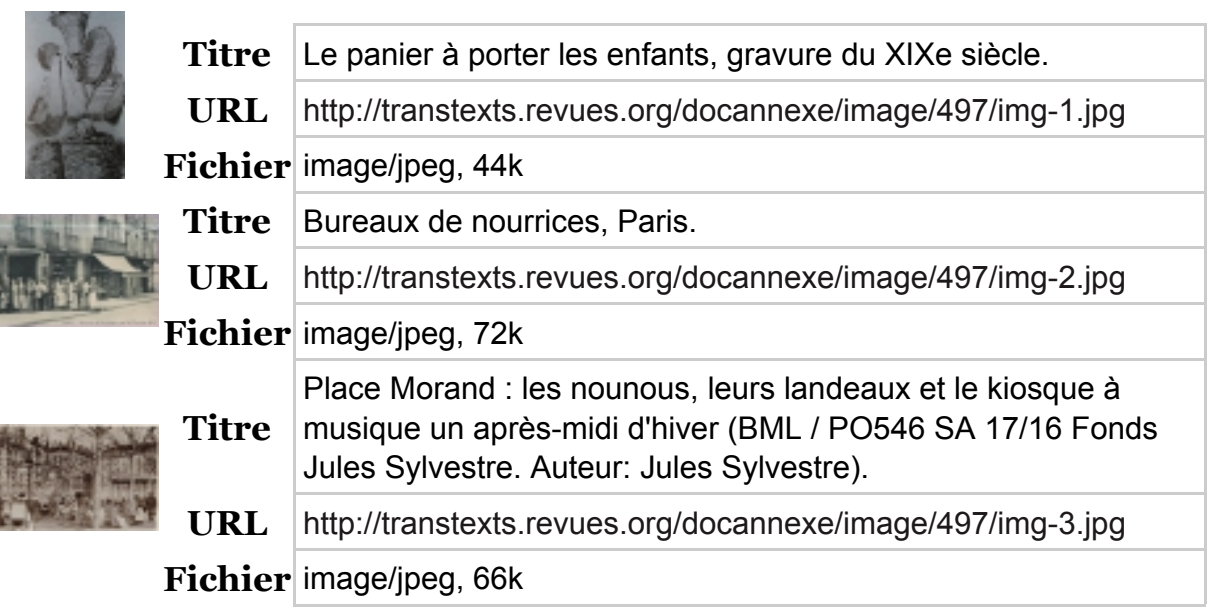

\section{Pour citer cet article}

Référence électronique 
Emmanuelle ROMANET, « La mise en nourrice, une pratique répandue en France au XIXe siècle », Transtext(e)s Transcultures 跨文本跨文化 [En ligne], 8 | 2013, mis en ligne le 02 décembre 2013, consulté le 15 janvier 2014. URL : http://transtexts.revues.org/497

\section{Auteur}

\section{Emmanuelle ROMANET}

Docteur en histoire, Emmanuelle Romanet est chercheur associé à l'IETT (EA 4186) Université Jean Moulin Lyon 3. Elle travaille sur la situation des femmes dans leurs relations au monde du travail et la situation des femmes dans leur rôle de mère, de l'Ancien Régime jusqu'au XIXe siècle.

Emmanuelle Romanet has a PHD in History and is an associate researcher at IETT (EA 4186), Universite Jean Moulin Lyon 3. She works on the condition of women in their relationships with the working world and in their role as mothers, spanning the period from the Ancien Régime to the 19th century.

\section{Droits d'auteur}

(c) Tous droits réservés 